\title{
Implicación de los profesionales de la salud en los casos de violencia en la pareja contra la mujer: La influencia de las actitudes sexistas hacia la mujer
}

\author{
Nancy Noriega ${ }^{1}$, Joel Juarros-Basterretxea ${ }^{2 *}$ y Juan Herrero ${ }^{3}$ \\ 'Servicio de Salud del Principado de Asturias (SESPA) (España) \\ 2Universidad de O'Higgins (Chile) \\ ${ }^{3}$ Universidad de Oviedo (España)
}

- Recibido: 09 - 05 - 2019 . Aceptado: 25 - 10 - 2019 . Avance Online: 14 - 11 - 2019

RESUMEN: Los profesionales sanitarios encuentran serias dificultades para identificar los casos de violencia en la pareja (IPV) contra la mujer. Las actitudes sexistas hacia la mujer y bajo nivel formativo en materia de IPV, pueden obstaculizan el abordaje de los casos de maltrato en los Servicios de Salud. Nuestro objetivo es analizar la relación entre actitudes sexistas y la implicación de los profesionales de la salud en los casos de IPV, así como el potencial efecto mediador de las horas de formación. Participaron 590 profesionales del Servicio de Salud del Principado de Asturias (España). Los resultados mostraron que ambos tipos de sexismo, -hostil y benevolente-, ejercen su influencia en la implicación en los casos de IPV. El sexismo hostil se relaciona con menores tasas de implicación, sin que las horas de formación medien esta relación. También mayores niveles de sexismo benevolente se relacionan con menores tasas de implicación, pero sólo mediado a través de las horas de formación.

PALABRAS CAVE: Sexismo hostil, Sexismo benevolente, Violencia en la pareja, Profesionales sanitarios, Implicación

Health professionals' involvement in cases of partner violence against women: The influence of sexist attitudes toward women

\begin{abstract}
Health professionals find it difficult to identify cases of partner violence (IPV) against women. Sexist attitudes toward women and low formation level on IPV can hinder the intervention on cases of IPV in Health Services. Our objective is to analyze the relationship between sexist attitudes and the implication of health professionals on IPV cases, as well as the potential mediating effect of training hours. 590 professionals from the Health Service of the Principality of Asturias (Spain) participated. The results showed that both types of sexism, hostile and benevolent, exert their influence on the involvement in cases of IPV. Hostile sexism is related to lower rates of involvement, without mediational effect of the hours of training. Higher levels of benevolent sexism are also related to lower rates of involvement, but only mediated by the hours of training.
\end{abstract}

KEY WORDS: Hostile sexism, Benevolent sexism, Intimate partner violence, Health professionals, Involvement

La violencia en la pareja íntima (IPV, del inglés intimate partner violence; violencia de género, [VG] de acuerdo con el marco legal español) contra la mujer es uno de los problemas

\footnotetext{
${ }^{*}$ Correspondence: Joel Juarros-Basterretxea.

Universidad de O'Higgins (Chile).

CP: 33003, Oviedo, España.
}

E-mail: juarrosbasterretxea.j@gmail.com

(c) 2020 Sociedad Universitaria de Investigación en Psicología y Salud. Publicado por Consejo General de Colegios Oficiales de Psicólogos, España. Este es un artículo Open Access

Citar como/Cite as: Noriega, $N$. Juarros-Basterretxea J y Herrera, J (2020) Implicación de los profesionales de la salud en los casos de violencia en la Implicación de los profesionales de la salud en los casos de violencia en la pareja contra la mujer: La inluencia de las actiludes sexistas hacia la mujer. Revista Iberoamericana de Psicología y Salud, 17 (1), 31-41. https://doi.org/10.23923/i. rips.2020.01.033 de salud que más graves en la sociedad actual. La comorbilidad asociada a la IPV hace que las mujeres que son víctimas de esta forma de violencia acudan a los Servicios de Salud con mayor frecuencia que la población general (Bonomi et al., 2009; Campbell, 2002; Montero et al., 2011 ). En este sentido, los profesionales de la salud juegan un papel central en la detección (y posterior intervención) sobre estos 
casos (Francis, Loxton, y James, 2016; GarcíaMoreno et al., 2015ab; MacMillan et al., 2009; Menéndez, Pérez, y Lorence, 2013; Soh, Grigg, Gurvich, Gavrilidis, y Kulkarni 2018). Sin embargo, la detección de casos de violencia de género es una tarea complicada para la cual los profesionales del ámbito sanitario no siempre están debidamente formados y que puede verse afectada por características individuales (p. e. actitudes, sesgos cognitivos).

Las dificultades de actuación propias de los casos de IPV hace necesario evaluar aquellos aspectos que puedan estar obstruyendo la detección de los mismos, ya que se encuentran limitaciones tanto a nivel individual, como organizacional y social (Djikanovic, Celik, Simic, Matejic, y Cucic, 2010; López-García, Ruiz-Hernández, Llor-Zaragoza, Llor-Zaragoza, y Jiménez-Barbero, 2018; Pérez-Fuentes, Molero-Jurado, Gázquez-Linares, y SimónPérez, 2019).

Uno de los aspectos más estudiados ha sido la formación (nivel organizacional) que reciben los profesionales de la salud con respecto a la violencia doméstica (VD) en general y la IPV en particular que cada vez está más presente en el plan de estudios de los profesionales de la salud (Hamberger, 2007). Se ha observado en varias investigaciones que el nivel de formación en materia de IPV es deficitaria y supone una de las principales limitaciones (en ocasiones indicada por los propios profesionales) en actuaciones tales como la detección de estos casos (véase Dijkanovic et al., 2010; Gutmanis, Beynon, Tutty, Wathen y MacMillan, 2007; Ramsay et al., 2012; Siendones et al., 2002; Soh et al., 2018; Sugg et al., 1999; Zink, Regan, Goldenhar, Pabst, y Rinto, 2004). Como se ha indicado anteriormente, son los propios profesionales de la salud los que dicen no tener la formación suficiente, lo que afecta a la percepción que tienen sobre sus capacidades para actuar en este tipo de casos. Esto hace que la intervención sobre los propios profesionales a fin de sobreponerse a este obstáculo, pues se ha comprobado la autoeficacia de los profesionales de la salud aumenta cuando se llevan a cabo programas de entrenamiento comprehensivo (Gadomski, Wolff, Tripp, Lewis, y Short, 2001). Sin embargo, algunos autores han indicado que intervenciones tales como los planes formativos son necesarios, pero insuficientes, y recomiendan el desarrollo de estructuras especializadas en IPV (p. e. grupos especializados o sistemas de vigilancia) que sirvan de complemento a la formación de los profesionales de la salud (Siendones et al., 2002).

La tendencia por parte de los propios profesionales a querer formarse y solicitar mayor conocimiento del fenómeno de la IPV muestra un claro cambio de actitud que queda patente en la comparación entre los resultados obtenidos en investigaciones de distintas épocas. A modo de ejemplo, en 1999 Sugg et al. observaron que tan tanto clínicos (50\%) como las enfermeras y asistentes (70\%) consideraban que la prevalencia de la IPV era mínima (menor o igual al $1 \%$ ) y en torno al $45 \%$ de los médicos nunca 0 rara vez preguntaban a sus pacientes durante el examen médico sobre la IPV. Por el contrario, once años después Dijkanovic et al. (2010) observaron tres temas centrales: (1) la percepción de la IPV, en la cual se englobó la concepción de la IPV como algo inaceptable, la comprensión de la víctima y una opinión crítica de la pareja; (2) la percepción de su propio rol frente a estos casos como agentes de apoyo de la víctima abiertos a colaborar con otras instituciones y fomentar la revelación de la violencia sufrida; y (3) las barreras para proveer ayuda adecuada a las víctimas como una red de apoyo débil, falta de educación y formación y la inseguridad social general. Las diferencias entre ambos estudios con apenas once años entre uno y otro indica que la implicación de los profesionales de este ámbito ha aumentado y con ello las tasas de detección que se acercan cada vez más a las de prevalencia. A pesar de esto, Dijkanovic et al. (2010) apuntan que sigue habiendo profesionales de este ámbito que muestran actitudes adversas (p. e. mostrarse interesados/desconfiados por las causas de la IPV) que sugieren una visión más tolerante hacia la IPV y potencialmente perjudicial de cara a que las víctimas revelen que lo son. Más recientemente, Soh et al. (2018) apoyaron con su estudio los resultados de Diikanovic et al. (2010) pues comprobaron que la mayoría de los participantes identificaban la IPV como 
un problema de salud, si bien apenas el 50\% se veía con mucha confianza a la hora de intervenir, poniendo de manifiesto las carencias formativas de estos profesionales sobre el tema.

Este cambio progresivo en cómo los propios profesionales de la salud ven esta forma de violencia y se ven a sí mismos con respecto a ella, muestra que, a pesar de que los aspectos organizacionales en relación a las actuaciones en casos de IPV han sido los que más interés científico han suscitado, también otros aspectos de carácter individual como las actitudes son relevantes. En este sentido, se ha observado que las actitudes (tanto por ser positivas como negativas) de los profesionales de la salud frente a este tipo de casos tienen un papel sumamente relevante (Diikanovic et al., 2010) y que las conductas prejuiciosas y discriminatorias influyen en la salud de los pacientes (véase por ejemplo Chrisler, Barney, y Palatino, 2016; Mouzon, Taylor, Woodward, y Chatters, 2017; Richman, Pascoe, y Lattaner, 2017), pero rara vez se han estudiado las actitudes sexistas hacia la mujer de estos profesionales, estrechamente relacionadas con la IPV, y su efecto sobre cómo abordan estos casos. En tanto que sesgo cognitivo, las actitudes sexistas hacia la mujer pueden resultar un aspecto fundamental de cómo se abordan los casos de IPV, pues se han relacionado con mayores niveles de aceptación y justificación de esta forma de violencia (Bringas-Molleda, Estrada-Pineda, Suárez-Álvarez, Torres, Rodríguez-Díaz, García-Cueto, \& RodríguezFranco, 2017).

Una de las teorías más influyentes sobre las actitudes sexistas hacia la mujer ha sido la Teoría del Sexismo Ambivalente de Glick y Fiske (1996) al contemplar, además de los característicos prejuicios hostiles hacia la mujer (sexismo hostil), aspectos subjetivamente positivos hacia ellas, pero que siguen siendo estereotípicos (sexismo benevolente). Esta teoría supone un gran avance en el concepto de sexismo hacia la mujer al abarcar tanto el rechazo que el sexismo tradicional implicaba como la tendencia por parte de los hombres a mantener relaciones estables e íntimas con ellas (Juarros-Basterretxea, Overall, Herrero, y Rodríguez-Díaz, 2019). Como se ha indicado anteriormente, las actitudes sexistas hacia la mujer en profesionales de la salud han sido escasamente estudiadas (véase McDonald, Terry, y Tehranifar, 2014; Richamn, Pascoe, y Lattaner, 2017, para una revisión), pero del mismo modo que otras formas de discriminación (p. e. racismo) se han relacionado con más problemas de salud en los pacientes, cabe pensar que actitudes sexistas hacia la mujer puedan estar relacionadas con deficiencias en la atención (menor nivel de implicación) de las pacientes víctimas IPV y la formación al respecto por parte de estos profesionales. Así, las actitudes sexistas hostiles se han relacionado con mayores tasas de aceptación y justificación (Herrero, Rodríguez, y Torres, 2017; Sakalli, 2001 ; Valor-Segura, Expósito, y Moya, 2011) de la IPV, lo que explicaría los casos de profesionales que muestran actitudes adversas (Dijkanovic et al., 2010) o la sensación de estar siendo juzgadas que refieren algunas pacientes (véase Feder, Hutson, Ramsay, y Taket, 2006). Por su parte, se ha observado que las actitudes sexistas benevolentes tienen un efecto protector frente a la IPV del hombre contra la mujer al asociarse con un mayor rechazo de la violencia (Allen, Swan, y Raghavan, 2009; Sakalli, 2001). Es importante tener en cuenta como bien indican Allen et al. (2009) que se observa una tendencia a proteger más a aquellas mujeres que se percibe que encajan con su "rol tradicional", por lo que la relación entre la aceptación y justificación de la IPV y el sexismo hostil y benevolente ha de analizarse teniendo en cuenta la relación entre ambas formas de sexismo. Tal y como indican Valor-Segura ef al. (2011), aunque la relación del sexismo benevolente con la tendencia a culpabilizar a la víctima desaparece al tener en cuenta el sexismo hostil, es la interacción de este último con el benevolente lo que mejor explica que se culpabilice a la víctima.

La relevancia del análisis de las actitudes sexistas hacia la mujer radica en su potencial rol como impulsor o freno a la hora de buscar formación en materia de detección e intervención en casos de IPV, así como en la falta de objetividad en la evaluación de los casos. Tal y como vienen indicando los estudios (Hamberger, 2007; Rojas, Gutiérrez, Alvarado, y Fernández, 2015) actitudes más positivas hacia el abordaje de los casos de 
IPV se relacionan con mayores niveles de formación y utilización de recursos. En este sentido, visiones sesgadas de los casos de IPV, ya sea por tener una mayor aceptación de esta forma de violencia, por justificarla, o por culpar a la víctima, puede propiciar que profesionales no busquen formarse en esta materia e incluso que no hagan un uso adecuado de los recursos de los que disponen. A pesar de todo, no se estudian los mecanismos por los cuales algunos profesionales buscan o solicitan ser formados en materia de IPV, mientras que otros no lo hacen e incluso continúan manifestando actitudes adversas al encontrarse ante casos de IPV.

Así pues, se pretende abordar en la presente investigación el papel que las actitudes sexistas ambivalentes (hostiles y benevolentes) juegan en la implicación de los profesionales sanitarios en los casos de IPV, considerando el potencial rol mediador de la formación en esta materia. Mediante un modelo de ecuaciones estructurales se van a analizar tanto los efectos directos como indirectos (mediados por la formación) de las actitudes sexistas sobre la implicación en la atención a las pacientes.

\section{MÉTODO}

\section{- PARTICIPANTES}

La muestra estuvo compuesta por 590 profesionales del Servicio de Salud del Principado de Asturias (SESPA) (España) de entre 23 y 64 años $(M=48.45, D E=9.52)$, de los cuales el 79.5\% ( $n=469)$ eran mujeres $y$ el $20.5 \%(n=121)$ hombres. El 7.8\% $(n=$ 48) se percibió de clase social media-baja, el $74.7 \%$ ( $n=457)$ de clase social media y el $16.8 \%(n=103)$ de clase social media- alta, mientras que sólo algunos casos se percibieron de clase social baja $(.2 \%, n=1)$ o alta $(.5 \%$, $n=3)$.

Con respecto a las características del puesto de trabajo, el $42.3 \%$ ( $n=259)$ de los participantes desarrollaban su trabajo en el ámbito de la atención primaria y el $57.7 \%$ ( $n=$ 353) en el ámbito de la atención especializada. El $31.1 \%(n=191)$ eran personal sanitario facultativo y el 67.8 ( $n=416)$ no facultativo, mientras que el $1.1 \%(n=7)$ restante pertenecían a otras categorías profesionales. El $71.2 \%$ ( $n=437)$ se encontraban en la situación de estatutario fijo, el $11.9 \%(n=73)$ de estatutario eventual, el $12.4 \%(n=76)$ de estatutario interino y el $4.6 \%(n=28)$ en otras situaciones.

\section{-VARIABLES DE MEDIDA}

\section{VARIABLE DE RESULTADO}

Implicación de los profesionales sanitarios. Se elaboró una escala ad hoc para valorar la implicación de los profesionales sanitarios en la detección de los casos de violencia en la pareja. Esta escala consta de cinco ítems ( $p$. e. "El profesional sanitario debería dedicar un esfuerzo adicional para identificar y detectar casos potenciales de violencia en la pareja"). Los ítems se respondían en una escala de tipo Likert con categorías de respuesta que oscilan entre 1 (totalmente en desacuerdo) a 5 (totalmente de acuerdo) ( $\alpha$ de Cronbach $=.85$ ).

\section{VARIABLE PREDICTORA}

Actitudes sexistas ambivalentes. Se utilizó la versión reducida del Inventario de Sexismo Ambivalente hacia mujeres (ASI) de Glick y Fiske (1996) (Rodríguez, Lameiras, y Carrera, 2009). El inventario consta de seis ítems que miden sexismo hostil (p. e. "Las mujeres exageran los problemas que tienen en el trabajo") y seis ítems que miden sexismo benevolente (p. e. "Las mujeres deben ser queridas y protegidas por los hombres"). Los doce ítems se respondían con una escala Likert de cinco puntos que oscilaba entre 1 (completamente en desacuerdo) y 5 (completamente de acuerdo). Las puntuaciones fueron sumadas para construir dos puntuaciones separadas sobre el nivel de sexismo hostil ( $\alpha=$ .87) y sexismo benevolente $(\alpha=.88)$.

\section{VARIABLE MEDIADORA}

Horas de formación en materia de violencia de género. El número de horas de formación en materia de violencia de género fue codificado en cinco categorías: 0 horas, entre 1 y 5 horas, entre 6 y 20 horas, entre 
21 y 60 horas y, por último, más de 60 horas $(M=.72, D E=1.19)$. El 68.8\% $(n=410)$ de los participantes no había recibido ninguna formación en materia de violencia de género, el $7.1 \%(n=42)$ entre 1 y 5 horas, el $12.4 \%$ $(n=73)$ entre 6 y 20 horas, el $8.1 \%(n=48)$ entre 21 y 60 horas y apenas un $3.9 \%(n=23)$ habían recibido más de 60 horas de formación.

\section{-PROCEDIMIENTO}

Previa aplicación del cuestionario se solicitó la autorización correspondiente a la Comisión de Docencia y Comisión de Investigación del Hospital del Valle Nalón, el Comité Ético de Investigación Clínica del Principado de Asturias, la Unidad de Calidad del Hospital del Hospital Valle Nalón, la Coordinación Asistencial y de Cuidados del Servicio de Salud del Principado de Asturias, la Gerencia del Área VIII. Una vez obtenida la aprobación y autorización para llevar a cabo la investigación se contó con la ayuda del Servicio de Informática del Hospital para la difusión del cuestionario a través del correo interno SESPA WEBMAIL con 3.730 usuarios, obteniendo una tasa de respuesta válida del 16.46\%.

Previa cumplimentación del cuestionario se informó a los participantes del carácter voluntario, anónimo y confidencial del estudio.

\section{-ANÁLISIS DE LOS DATOS}

Se calculó un modelo de ecuaciones estructurales para el análisis del ajuste del modelo a los datos y efecto total, directo e indirecto de las actitudes sexistas hostiles y benevolentes sobre la implicación de los profesionales de la salud en los casos de violencia en la pareja cuando se tenía en cuenta la relación entre ambas formas de sexismo y el rol mediador de las horas de formación en materia de violencia de género. Posteriormente, el mismo modelo fue calculado teniendo en cuenta la edad, el sexo y la clase social percibida como covariables.

El análisis descriptivo de los datos se realizó mediante el paquete estadístico IBM SPSS Statistics 22, el programa y la estimación del modelo de ecuaciones estructurales se realizó mediante el programa estadístico EQS 6.3 (Bentler, 1995).

\section{RESULTADOS}

En la tabla 1 se muestran los estadísticos descriptivos y las correlaciones entre las variables. Tal y como se esperaba, el sexismo hostil y benevolente están significativa y positivamente relacionados entre sí $(r=.471$, $p \leq .01$ ). Además, tanto el sexismo hostil como el sexismo benevolente se relacionan de forma significativa y negativa con la implicación en los casos de violencia en la pareja $(r=-.290$, $p \leq .01 ; r=-.161, p \leq .01$ respectivamente) y con las horas de formación en esta materia $(r=-.188, p \leq .01 ; r=-.203, p \leq .01$, respectivamente). Las horas de formación de relacionan significativa y positivamente con la implicación en los casos de violencia en la pareja $(r=.199, p \leq .01)$.

Tabla 1

Correlaciones de Pearson, medias y desviaciones estándar de todas las variables del estudio

\begin{tabular}{|c|c|c|c|c|}
\hline Variables & 1 & 2 & 3 & 4 \\
\hline 1. Implicación & - & & & \\
\hline 2. Horas de formación & $.199^{\star *}$ & - & - & \\
\hline 3. Sexismo hostil & $-.290^{\star *}$ & $-.188^{\star *}$ & $.471^{* \star}$ & - \\
\hline 4. Sexismo benevolente & $-.161^{\star *}$ & $-.203^{* \star}$ & 13.69 & 14.74 \\
\hline$M$ & 21.51 & .72 & 6.28 & 6.48 \\
\hline$D E$ & 2.34 & 1.19 & & \\
\hline
\end{tabular}

Nota: ${ }^{\star} p \leq .05 ;{ }^{* \star} p \leq .01$. 
Los índices de ajuste obtenidos en el cálculo del modelo causal indican que el modelo presenta tiene un ajuste adecuado a los datos $\left(S-B_{\chi} 2=285.2625, g l=130\right.$, $\mathrm{p} \leq .001, \mathrm{CFI}=.95$, RMSEA $=.04$ I.C. al $95 \%[.04, .05])$. El efecto total $(\beta=-.364$, $p \leq .001)$ y directo $(\beta=-.351, p \leq .001)$, pero no el indirecto $(\beta=-.064, n s$.$) , de las$ actitudes sexistas hostiles sobre la implicación en los casos de IPV fueron significativos. En otras palabras, mayores niveles de sexismo hostil no se asociaron con el número de horas de formación en materia de IPV, pero si con menores niveles de implicación en casos de IPV. Estos efectos se calcularon teniendo en cuenta la relación entre el sexismo hostil y el benevolente $(r=.562, p \leq .01)$. Por su parte, sólo el efecto indirecto (a través de las horas de formación) del sexismo benevolente sobre la implicación fue significativo $(\beta=-.172, p \leq$ $.001)$, mientras que el efecto directo $(\beta=.003$, ns) y el total ( $\beta=-.031, n s)$ no lo fueron.

El modelo explica el $18.4 \%$ de la varianza de las actitudes hacia el tratamiento, y predice que las puntuaciones en sexismo hostil se relacionan con actitudes más negativas hacia el tratamiento, independientemente del nivel de formación en violencia de género. Por otra parte, el sexismo benevolente influirá en las actitudes hacia el tratamiento, en la medida en que afecte negativamente a la formación en violencia de género.

\section{DISCUSIÓN}

Utilizando los datos de 590 profesionales del Servicio de Salud del Principado de Asturias (España), en el presente estudio se examinan las asociaciones entre las actitudes sexistas ambivalentes (hostiles y benevolentes) y la implicación de en los casos de violencia en la pareja íntima (IPV). El objetivo fue analizar el efecto que las actitudes sexistas hostiles y benevolentes hacia la mujer tienen en la implicación de los profesionales de la salud en los casos de IPV, teniendo en cuenta el potencial rol mediador de las horas de formación que sobre esta materia reciben dichos profesionales.

Los resultados obtenidos en la presente investigación indican que tanto las actitudes sexistas hostiles como benevolentes de los profesionales de la salud se relacionan con los niveles de implicación que estos tienen en los casos de IPV, si bien de forma diferencial. En primer lugar, los resultados apoyan la hipótesis de que actitudes sexistas hostiles se relacionarán con menores niveles de implicación en casos de IPV. Así, aquellos participantes con mayores niveles de sexismo hostil mostraron también menor implicación en el abordaje de este tipo de casos. Por otra parte, los resultados también apoyan la hipótesis de que las actitudes sexistas benevolentes, a diferencia de las hostiles, se relacionarán de forma indirecta con los niveles de implicación. En este sentido, los

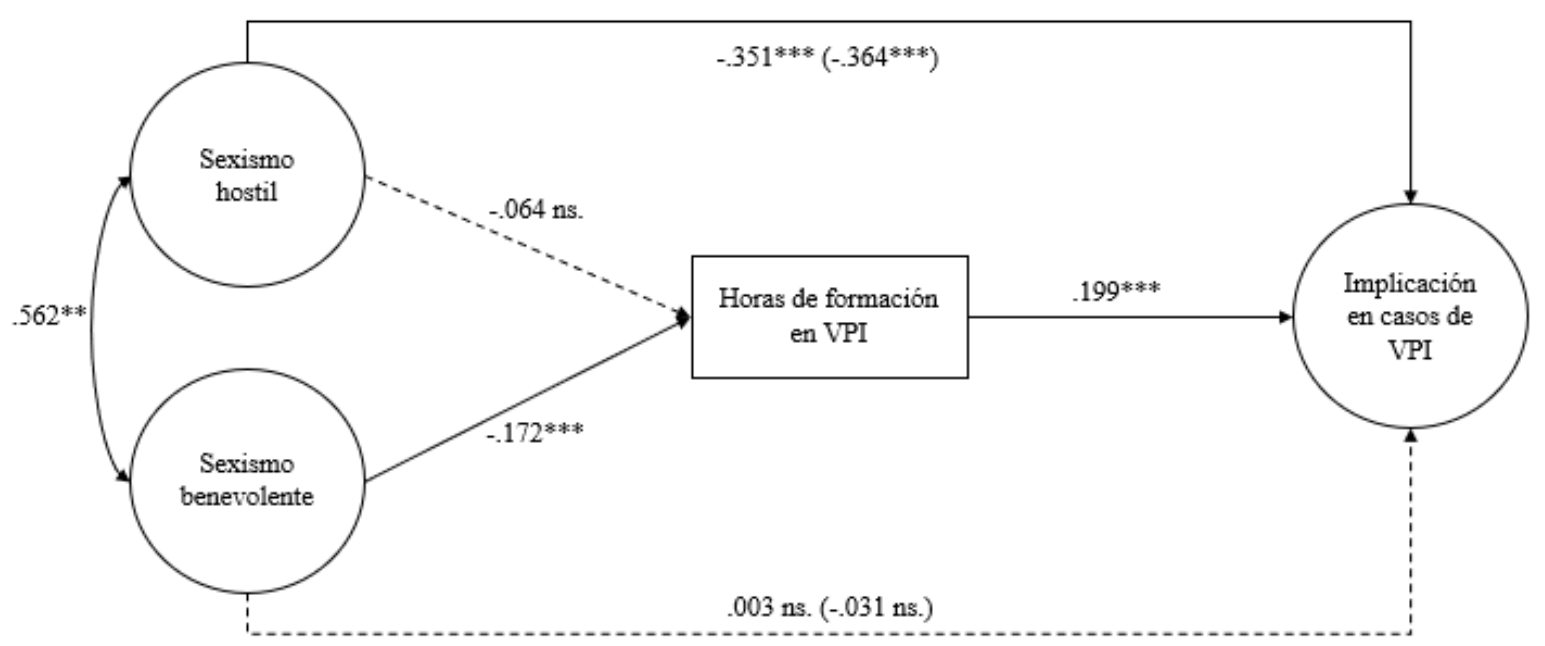

Figura 1: Efectos totales, directos e indirecto de las actitudes sexistas ambivalentes sobre la implicación en los casos de IPV considerando el efecto mediador de las horas de formación en materia de IPV. Los efectos totales están entre paréntesis $(N=590)$.

Nota: los ítems han sido suprimidos en la figura para su simplificación; ${ }^{*} p \leq .05 ;^{* *} p \leq .01{ }^{* * *} p \leq .001$. 
participantes con mayores niveles de sexismo benevolente referían una menor tendencia a formarse, si bien cuando se formaban los niveles de implicación aumentaban.

Tal y como han venido indicando distintos autores (Sakalli, 2001; Valor-Segura et al., 2011 ), el sexismo hostil hacia las mujeres se ha relacionado mayores niveles de aceptación de esta forma de violencia y tienen de culpabilizar a la víctima. Aplicado al ámbito concreto de la sanidad, se ha comprobado que las actitudes prejuiciosas o discriminatorias hacia los pacientes tienen efectos nocivos para la salud de estos (Chrisler et al., 2016; Mouzon et al., 2017; Richman et al., 2017), probablemente relacionado con la atención deficitaria derivada del prejuicio. Así, los resultados obtenidos en algunas investigaciones sobre actitudes adversas de los profesionales de la salud hacia las víctimas de IPV (Dijkanovic et al., 2010; Feder et al., 2006) podrían explicarse por la presencia de actitudes prejuiciosas hacia las mujeres (sexismo hostil). Estas actitudes se traducen en menores niveles de implicación en los casos de IPV y en una atención deficitaria, afectando a la salud de las mujeres víctimas de esta forma de violencia.

Por su parte, el sexismo benevolente, aunque relacionado con el hostil, se ha relacionado con actitudes más desfavorables hacia la IPV e incluso se ha hablado de cierto aspecto protector (Allen et al., 2009; Sakalli, 2001). En esta misma línea, cabría esperar que este rechazo se tradujese en el ámbito concreto de la atención sanitaria en mayores niveles de implicación en la atención a las víctimas de IPV, pero los resultados indican que esta forma de sexismo no se relaciona de forma directa con la implicación de los profesionales de la salud. Tal y como apuntan Allen et al. (2009), las actitudes sexistas benevolentes guardan una particular relación con la IPV; si bien es cierto que se observa una menor aceptación de esta forma de violencia en aquellos sujetos con mayores niveles de sexismo benevolente, también se ha comprobado que esto se relaciona con el hecho de que las mujeres se amolden a la visión estereotípica de la mujer, tendiendo a justificar la violencia ejercida sobre ellas cuando no es así. Así pues, la ausencia de relación del sexismo benevolente con la implicación (en este caso) podría deberse al hecho de que, por una parte, el sexismo benevolente no es suficiente para explicar el rechazo o justificación de violencia y por otra, se desconocen los detalles específicos (si la mujer se amolda o no a lo que se espera de ella). Sin embargo, si se observa una relación indirecta entre las actitudes sexistas benevolentes y la implicación a través del número de horas de formación recibidas en materia de IPV. Por una parte, los mayores niveles de sexismo ambivalente se relacionan con una tendencia a recibir menos horas de formación, pero, por otra, se observa que, una vez recibida la formación, el mayor número de horas se relaciona con mayores niveles de implicación. El papel del sexismo benevolente es, pues, especialmente relevante en el efecto que tiene sobre la tendencia a formarse (más o menos) o no formarse en materia de IPV, actuando como un proceso de autoselección que va a determinar los futuros niveles de implicación. Los hallazgos de diferentes estudios resaltan el impacto significativo que tienen la capacitación y la formación con mejores tasas de detección (Gutmanis et al. 2007; Macías, 2012; Yeung, Chowdhury, Malpass y Feder, 2012). La escasez de registros dificulta la constatación del modo en que una práctica formativa puede provocar un cambio en las actitudes de los sanitarios y, si este cambio verdaderamente incrementa o no la detección, o sí precisa de acciones formativas continuadas en el tiempo.

Tal y como han indicado algunos autores, más relevante que la relación de ambas formas de sexismo por separado con la aceptación de la IPV, es la consideración conjunta de ambas, pues se ha comprobado la relación entre el sexismo benevolente y la aceptación desaparece en estos casos, pero es lo que mejor predice la aceptación (Valor-Segura et al., 2011). La relación entre ambas formas de sexismo y su influencia sobre los niveles de implicación de los profesionales de la salud en los casos de IPV tiene repercusiones a nivel social, pues condiciona la detección (infra-detección) de los casos de IPV en uno de los ámbitos a los que las víctimas de esta forma de violencia acuden principalmente. Estos resultados sugieren que la presencia de actitudes sexistas 
tanto hostiles como benevolentes, podrían obstaculizar la percepción de las situaciones de este tipo de maltrato, como sugieren algunos estudios (Masser, Lee y Mclnmmie, 2010; Napier, Thorisdottir y Jost, 2010) y con ello, la comprensión y el tratamiento de los casos de IPV. Estos datos, estarían en la misma línea de algunos estudios que confirman la relevancia de determinadas actitudes de género en la evaluación que hacen de una mujer que ha sido víctima de violencia de pareja (Abrams, Viki, Masser, y Bohner, 2003; Masser et al., 2010; Soto-Quevedo, 2012; Herrera, Herre, y Expósit0, 2018).

$\mathrm{Ha}$ de tenerse en cuenta que le cambio actitudinal no depende exclusivamente de la formación. Tal y como indicaron Edwardsen, Horwitz, Pless, Le Roux y Fiscella (2011), la formación por sí sola no supone una actitud positiva hacia la detección de casos a lo largo del tiempo, sino que son necesarias diversas medidas para que esto ocurra. De este modo, no es posible concretar el nivel formativo que garantice un cambio positivo en las actitudes profesionales hacia la mujer víctima de maltrato. En resumen, la formación que fomente en cambio hacia actitudes positivas (y mayores niveles de implicación) hacia la detección de casos de IPV es necesaria, pero no suficiente para afrontar la complejidad de estos casos (véase Siendones et al., 2002). Las actitudes sexistas son uno de los aspectos sobre los cuales trabajar a fin de evitar una infradetección de casos de IPV derivado de mayores niveles de aceptación y justificación de esta forma de violencia.

El presente estudio cuenta con distintas limitaciones y fortalezas. En primer lugar, la muestra obtenida para este estudio no es representativa de la totalidad de profesionales del Servicio de Salud del Principado de Asturias, ya que a pesar de que el cuestionario ha sido enviado a todos los profesionales del SESPA la tasa de respuesta ha sido muy baja (16.46\%). La falta de representatividad de la muestra limita la generalización de los resultados, pero cuenta con la ventaja de no tratarse de una muestra de conveniencia y que es lo suficientemente amplía para cumplir con los requisitos estadísticos requeridos para los análisis realizados.
Además, otros aspectos no contemplados en esta investigación pueden estar también explicando la falta de implicación más allá de las actitudes sexistas. Muy especialmente, elementos individuales como el propio burnout de los profesionales de salud (Pérez-Fuentes et al., 2019) o incluso el temor a represalias por parte de la pareja violenta (López-García et al., 2018), se deberían también tener en cuenta en futuras investigaciones.

A pesar de las limitaciones planteadas, los resultados obtenidos en la presente investigación suponen un avance relevante en lo referente a la influencia de las actitudes sexistas de los profesionales de la salud sobre su implicación. Aunque los efectos de la discriminación han sido ampliamente estudiados en otros casos, los investigadores se han centrado mayoritariamente en el estudio del racismo, no siendo hasta épocas más recientes cuando el sexismo ha empezado a cobrar importancia (Richman et al., 2017). La falta de estudios sobre este tema hace necesario seguir profundizando en tipos específicos que permitan un mayor conocimiento y entendimiento del efecto que las actitudes sexistas tienen sobre el abordaje estos casos por parte de los profesionales de la salud y de los cuales se deriven recomendaciones prácticas para los procesos de formación específica en la detección de casos de IPV. En este sentido, cabe destacar la pertinencia de centrar la evaluación en los propios profesionales de la salud para evaluar sus sesgos cognitivos en conjunto con las percepciones de los pacientes que ya ha sido estudiadas en algunos casos (Richman et al., 2017).

- Conflicto de intereses.

Los autores declaran no tener ningún conflicto de intereses.

\section{REFERENCIAS}

Abrams, D., Viki, G. T., Masser, B., y Bohner, G. (2003). Perception of a stranger and acquaintance rape: The role of benevolent and hostile sexism in victim blame and rape proclivity. Journal of Personality and Social Psychology, 84,111-125. https://doi.org/10.1037/00223514.84 .1 .111 
Allen, C. T., Swan, S. C., y Raghavan, C. (2009). Gender symmetry, sexism, and intimate partner violence. Journal of Interpersonal Violence, 24, 1816-1834. https://doi. org/10.1177/0886260508325496

Bentler, P. M. (1995). Structural equations program manual. Encino, CA: Multivariate Software, Inc.

Bonomi, A. E., Anderson, M. L., Reid, R. J., Rivara, F. P., Carrell, D., y Thompson, R. S. (2009). Medical and psychosocial diagnoses in women with a history of intimate partner violence. Archives of International Medicine 169, 1692-1697. http://dx.doi.org/10.1001/ archinternmed.2009.292

Bringas Molleda, C., Estrada Pineda, C., Suárez Álvarez, J., Torres, A., Rodríguez Díaz, F. J., García Cueto, E., \& Rodríguez Franco, L. (2017). Actitud sexista y trascendente durante el noviazgo entre universitarios latinoamericanos. Revista Iberoamericana de Psicología y Salud, 8 (1), 44-55.

Campbell, J. C. (2002). Health consequences of intimate partner violence. The Lancet, 359, 1331-1336. https://doi.org/10.1016/S01406736(02)08336-8

Chrisler, J. C., Barney, A., y Palatino, B. (2016). Ageism can be hazardous to women's health: Ageism, sexism, and stereotypes of olderwomen in the healthcare system. Journal of Social Issues, 72, 86-104. https://doi.org/10.1111/ josi. 12157

Diikanovic, B., Celik, H., Simic, S., y Cucic, V. (2010). Health professionals' perceptions of intimate partner violence against women in Serbia: Opportunities and barriers for response improvement. Patient Education and Counseling, 80, 88-93. https://doi. org/10.1016/i.pec.2009.09.028

Edwardsen, E. A., Horwitz, S. H., Pless, N. A. le Roux, H. D., y Fiscella, K. A. (2011). Improving identification and management of partner violence: examining the process of academic detailing: A qualitative study. BMC Medical Education, 11, 36. http://dx.doi. org/10.1186/1472-6920-11-36

Feder, G. S., Hutson, M., Ramsay, J., y Taket, A. R. (2006). Women exposed to intimate partner violence. Expectations and experiences when they encounter health care professionals: A meta-analysis of qualitative studies. Archives of International Medicine, 166, 22-37. Doi: 10.1001/archinte.166.1.22

Francis, L., Loxton, D., y James, C. (2016). The culture of pretence: a hidden barrier to recognizing, disclosing, and ending domestic violence. Journal of Clinical Nursing, 26, 2202-2214. https://doi.org/10.1111/ jocn. 13501

Gadomski, A. M., Wolff, D., Tripp, M., Lewis, C., y Short, L. M. (2001). Changes in health care providers' knowledge, attitudes, beliefs, and behaviors regarding domestic violence, following a multifaceted intervention. Academic Medicine, 76, 1045-1052. Recuperado de https://journals.Iww.com/ academicmedicine/Fulltext/2001/10000/ Changes in Health_Care Providers Knowledge, . 15.aspx\#pdf-link

García-Moreno, C., Hegarty, K., Lucas d'Oliveira, A. F., Koziol-McLain, J., Colombini, M., y Feder, G. (2015a). The health-systems response to violence against women. The Lancet, 385, 1567-1579. https://doi. org/10.1016/s0140-6736(14)61837-7

García-Moreno, C., Zimmerman, C., MorrisGehring, A., Heise, L., Amin, A., Montoya, O., Bathe-Deosthali, P., Kilonzo, N., y Watts, C. (2015b). Addressing violence against women: A call to action. The Lancet, 285, 1685-1695. https://doi.org/10.1016/ S0140-6736(14)61830-4

Glick, P., y Fiske, S. T. (1996). The Ambivalent Sexism Inventory: Differentiating hostile and benevolent sexism. Journal of Personality and Social Psychology, 70, 491-512. http:// dx.doi.org/10.1037/0022-3514.70.3.491

Gutmanis, I., Beynon., Tutty, L., Wathen,C. N., y MacMillan, H. L.(2007). Factors influencing identification of and response to intimate partner violence: a survey of physicians and nurses. BMC Public Health, 7, 12-23. http:// dx.doi.org/10.1186/1471-2458-7-12.

Hamberger, L. K. (2007). Preparing the next generation of physicians: Medical school and residency-based intimate partner violence curriculum and evaluation. Trauma 
Violence Abuse, 8, 214-225. https://doi. org/10.1177/1524838007301163

Herrera, M. C., Herrera, A., y Expósito, F. (2018).

To confront versus not to confront: Women's perception of sexual harassment. European Journal of Psychology Applied to Legal Context, 10, 1-7. https://doi.org/10.1016/i. ejpal.2017.04.002

Herrero, J., Rodríguez, F. J., \& Torres, A. (2017). Acceptability of partner violence in 51 societies: The role of sexism and attitudes toward violence in social relationships. Violence against women, 23(3), 351-367.

Juarros-Basterretxea, J., Overall, N., Herrero, J., \& Rodríguez-Díaz, F. J. (2019). Considering the Effect of Sexism on Psychological Intimate Partner Violence: A Study with Imprisoned Men. European Journal of Psychology Applied to Legal Context, 17 (2).

López-García, C., Ruiz-Hernández, J. A., LlorZaragoza, L., Llor-Zaragoza, P., y JiménezBarbero, J. A. (2018). User violence and psychological well-being in primary health-care professionals. European Journal of Psychology Applied to Legal Context, 10(2), 57-63. https:// doi.org/10.5093/ejpalc2018a6

Macías, J., Gil, E., Rodríguez, M. A., González, J. R., González, M. M., y Soler, A. M. (2012). Creencias y actitudes del alumnado de enfermería sobre la violencia de género. Index de Enfermería, 21, 9-13. http://dx.doi. org/10.4321/S1132-12962012000100003

MacMillan, H. L., Wathen, C. N., Boyle, M. H., Shannon, H. S., Ford-Gilboe, M., Worster, A., ..., y McNutt, L. A. (2016). Screening for intimate partner violence in health care settings. A randomized trial. Journal of the American Medical Association, 302, 493-501. http:// dx.doi.org/10.1001/jama.2009.1089

Masser, B., Lee, K., y McKimmie, B. M. (2010). Bad woman, bad victim? Disentangling the effects of victim stereotypicality, gender stereotypicality and benevolent sexism on acquaintance rape victim blame. Sex Roles, 62,494-504. http:// dx.doi.org/10.1007/s 11 199-009-9648-y

McDonald, J. A., Terry, M. B., y Tehranifar, P. (2014). Racial and gender discrimination, early life factors, and chronic physical health conditions in midlife. Womens' Health Issues, 24, 53-59. https://doi.org/10.1016/i.whi.2013.09.006

Menéndez, S., Pérez, J., y Lorence, B. (2013) La violencia de pareja contra la mujer en España: Cuantificación y caracterización del problema, las víctimas, los agresores y el contexto social y profesional. Psychosocial Intervention, 22, 4153. https://doi.org/10.5093/in2013a6

Montero, I., Ruiz-Pérez, I., Martín-Baena, D., Talavera, M., Escribá-Aguir, V., y Vives, C. (2011). Violence against women from different relationship contexts and health care utilization in Spain. Women's Health Issues, 21, 400-406. https://doi.org/10.1016/i.whi.2011.04.003

Mouzon, D. M., Taylor, R. J., Woodward, A., y Chatters, L. M. (2017). Everyday racial discrimination, everyday non-racial discrimination, and physical health among African Americans. Journal of Ethnic \& Cultural Diversity in Social Work, 26, 68-80. http:// dx.doi.org/10.1080/15313204.2016.11871 $\underline{03}$

Napier, J. L., Thorisdottir, H., y Jost, J. T. (2010). The joy of sexism? A multinational investigation of hostile and benevolent justifications for gender inequality and their relations to subjective wellbeing. Sex Roles, 62, 405-419. http:// dx.doi.org/10.1007/s $11199-009-9712-7$.

Pérez-Fuentes, M. C., Molero-Jurado, M. M., Gázquez-Linares, J. J., y Simón-Pérez, M. M. (2019). Analysis of burnout predictors in nursing: Risk and protective psychological factors. European Journal of Psychology Applied to Legal Context, 17 (1), 33-40. https:// doi.org/10.5093/eipalc2018a13

Ramsay, J., Rutterford, C., Gregory, A., Dunne, D., Eldridge, S., Sharp, D., y Feder, G. (2012). Domestic violence: knowledge, attitudes, and clinical practice of selected UK primary healthcare clinicians. British Journal of General Practice, 62, 647-655. http://dx.doi. org/10.3399/bigp 1 2X654623

Richman, L. S., Pascoe, E., y Lattaner, M. (2017). Interpersonal discrimination and physical health. En B. Major, J. F. Dovido, y B. G. Link (Eds.), The Oxford handbook of stigma, discrimination, and health. New York: Oxford University Press. 
Rodríguez, Y., Lameiras, M., y Carrera, M. V. (2009). Validación de la versión reducida de las escalas ASI y AMI en una muestra de estudiantes españoles. Psicogente, 12, 28429. Recuperado de https://dialnet.unirioja.es/ descarga/articulo/3265018.pdf

Rojas, K., Gutiérrez, T., Alvarado, R., y Fernández, A. (2015). Actitud hacia la violencia de género de los profesionales de atención primaria: Estudio comparativo entre Cataluña y Costa Rica. Aten Primaria, 47, 490-497. https://doi. org/10.1016/i.aprim.2014.10.008

Sakalli, N. (2001). Beliefs about wife beating among Turkish college students: The effects of patriarchy, sexism and sex differences. Sex Roles, 44, 599-610. http://dx.doi. org/10.1023/A: 1012295109711

Siendones R., Perea-Milla E., Arjona J. L., Agüera, C., Rubio, A., y Molina, M. (2002). Violencia doméstica y profesionales sanitarios: conocimientos, opiniones y barreras para la infradetección. Emergencias, 14, 224 232. Recuperado de http://emergencias. portalsemes.org/descargar/violencia-

domestica-y-profesionales-sanitariosconocimientos-opiniones-y-barreras-para-lainfradeteccion/force download/

Soh, H. J., Grigg, J., Gurvih, C., Gavrilidis, E., y Kulkarni, J. (2018). Family violence: An insight into perspectives of Australian health practitioners. Journal of Interpersonal Violence. Advance online publication. https://doi. org/10.1177/0886260518760609
Soto-Quevedo, O. A. (2012). Rol del sexismo ambivalente y de la transgresión de estereotipo de género en la atribución de culpa a mujeres víctimas de violencia de pareja. Acta Colombiana de Psicología, 15, 135-147. Recuperado de https://editorial.ucatolica.edu. co/ojsucatolica/revistas_ucatolica/index.php/ acta-colombiana-psicologia/article/view/273/ pdf\%20art\%C3\%ADculo\%2012

Sugg, N. K., Thompson, R. S., Thompson, D. C., Maiuro, R., y Rivara F. P. (1999). Domestic violence and primary care: Attitudes, practices, and beliefs. Archieves of Family Medicine, 8, 301-306. http://dx.doi.org/10.1001/ archfami.8.4.30

Yeung, H., Chowdhury, N., Malpass, A., y Feder, G. (2012). Responding to domestic violence in general practice: A qualitative study on perceptions and experiences. International. Journal of Family Medicine. http://dx.doi. org/10.1155/2012/960523

Zink, T., Regan, S., Goldenhar, L., Pabst, S., y Rinto, B. (2004). Intimate partner violence: What are physicians' perceptions? Journal of the American Board of Family Medicine, 7, 332-340. http://dx.doi.org/10.3122/ jabfm. 17.5.332 